\title{
PHOTON SCIENCE FOR RENEWABLE ENERGY
}

at Light-Source Facilities of Today and Tomorrow 


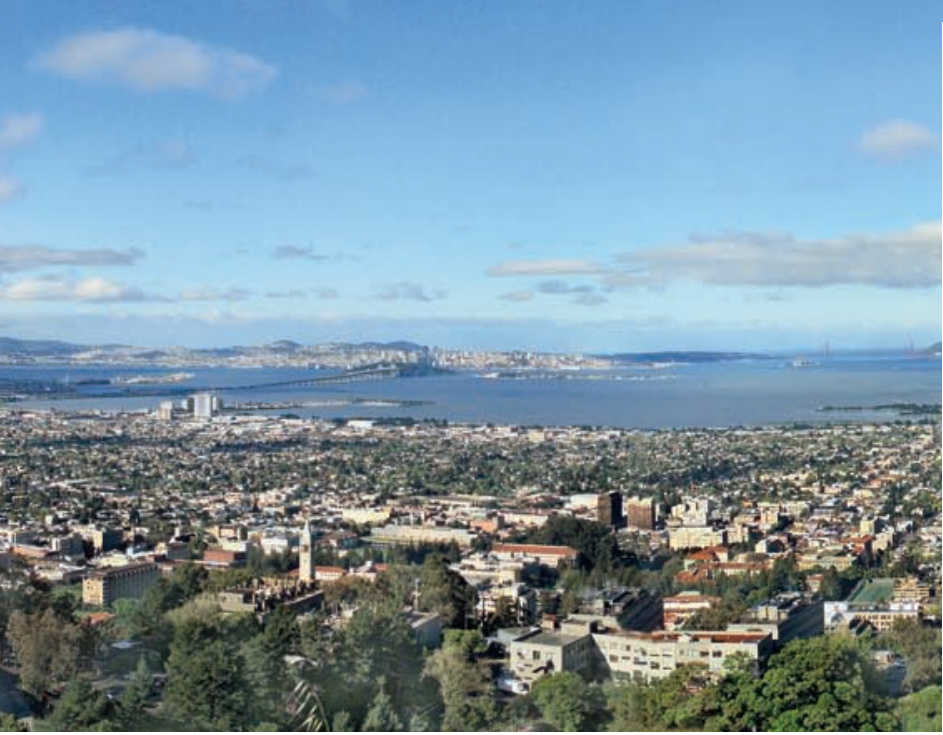

\section{FUNDAMENTAL QUESTIONS}

To establish the scientific foundations for the kind of transformative breakthroughs needed to build a 21st-century energy economy, we must address fundamental questions involving matter and energy. At right is a sampling of such questions that can be addressed by light-source facilities:

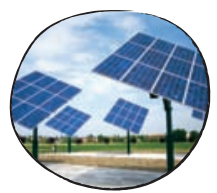

How can we modify the behavior of electrons and holes in semiconductors to more efficiently convert solar energy into electricity using earthabundant materials?

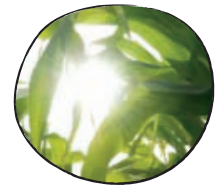

How can we harness photosynthesis and efficiently transform abundant plant material into biofuel? Can we produce fuel directly from sunlight by developing artificial photosynthesis?

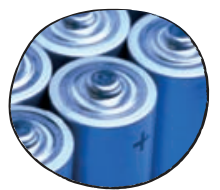

How can we increase the energy capacity and durability of lithium-ion batteries to maintain performance over hundreds to thousands of chargedischarge cycles?

How can we understand the electrical and chemical properties of a working electrochemical fuel cell to tailor its properties for the production of fuel or electricity?

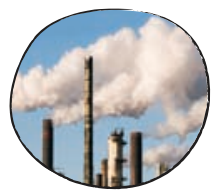

How does carbon dioxide interact with naturally occurring materials under ambient conditions during storage, and what can we learn about these materials to improve their capture capacity?

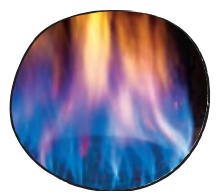

How can we identify combustion products at the parts-per-million level to improve efficiency and control pollution that results from the burning of fuel?

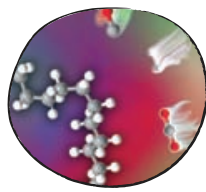

How do we tailor the electronic properties of nanostructured catalysts to achieve higher activity or selectivity with inexpensive materials? 


\section{ABOUT LIGHT SOURCES: SYNCHROTRON RADIATION AND FREE-ELECTRON LASERS}

Synchrotron radiation is emitted by electrons that are accelerated, generally in bunches through bending magnets around a storage ring with a circumference ranging from few hundred meters to a kilometer, to near the speed of light. This synchrotron "light," which may be a million times more intense than that produced by $x$-ray tubes, covers the spectrum from infrared to ultraviolet and from soft (long-wavelength) $x$ rays to hard (short-wavelength) $x$ rays. With the use of special multipole magnetic devices known as wigglers and undulators, the brightness of the emitted radiation can be increased to achieve high resolving power for spectroscopy/spectrometry, high spatial resolution for microscopy/spectromicroscopy/microtomography, and high coherence for diffraction/scattering.

The radiation emitted by a synchrotron storage ring scales with the number of electrons $(n)$ per bunch for a bending magnet, $\mathrm{nN}$ for a wiggler, and $\mathrm{nN}^{2}$ for an undulator ( $\mathrm{N}$ is the number of magnetic poles in the wiggler or undulator). Recent developments in linear accelerator technology using free-electron lasers have decreased the size of the electron bunches enough to emit coherent radiation in the soft to hard x-ray range with intensities that scale as $n^{2} \mathrm{~N}^{2}$, increasing brightness by ten orders of magnitude and timing resolution by five orders of magnitude.
Sunlight to electricity

Sunlight to fuel

Batteries

Fuel cells

$\mathrm{CO}_{2}$ capture \& sequestration

Combustion

Catalysis

A survey of beamlines that have performed energyrelated research at the Advanced Light Source (ALS) as of February 2010.

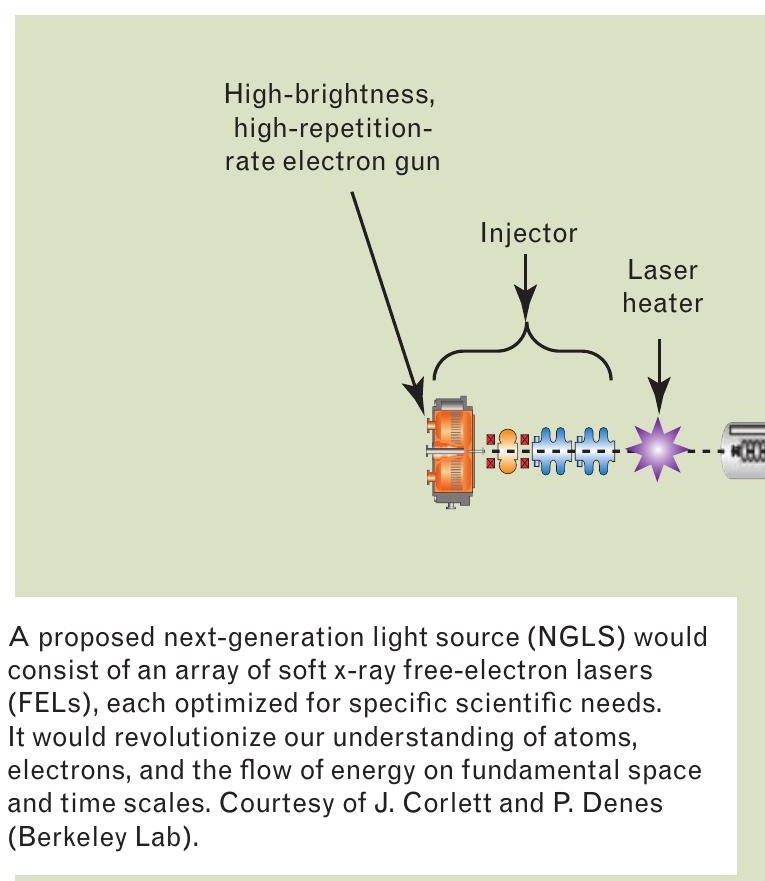




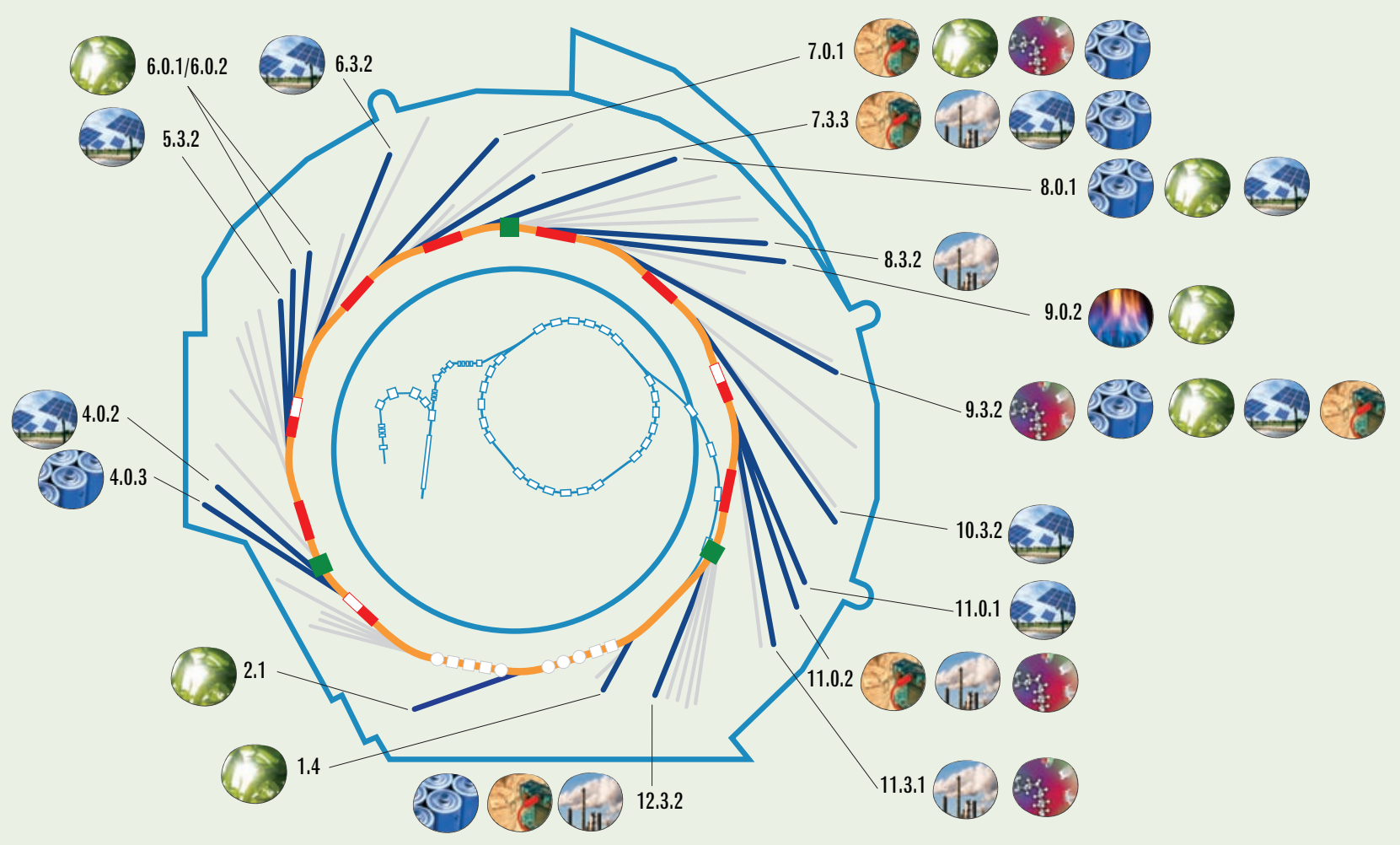

Harmonic

linearizer
Superconducting linac

(Linac 2)

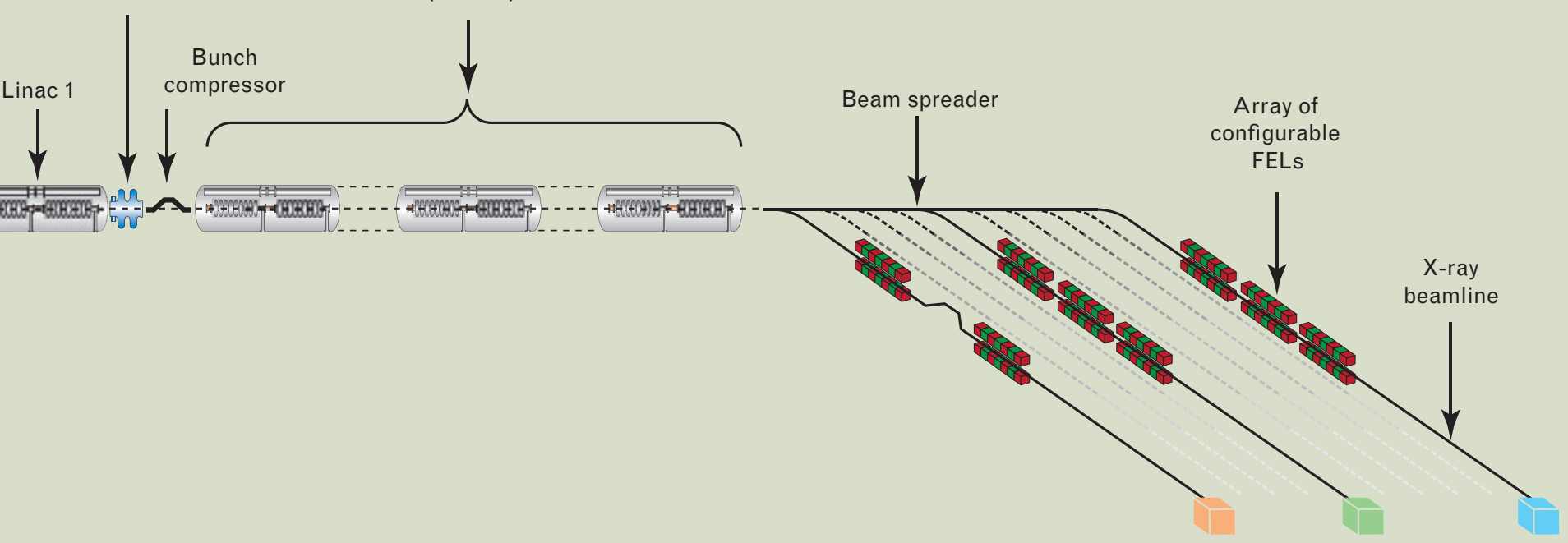

Endstations 


\section{SUNLIGHT TO ELECTRICITY}

The Sun delivers to Earth in one hour the amount of energy consumed globally in one year. However, solar power supplies just $1.5 \%$ of the world's demand, due to its higher cost relative to fossil fuels. To become competitive, rapid advances in converting solar energy into electricity are needed in order to produce lowcost, high-yield, and high-efficiency solar cells.

Metal impurities are among the main culprits in degrading the efficiency of multicrystalline solar cells. Scientists have combined $\mathrm{x}$-ray emission (fluorescence) and absorption spectroscopy to study the distribution, chemical state, and electron-hole recombination activity of metal clusters in a variety of multicrystalline solar cells before and after processing. They discovered that the size, distribution, and chemical binding of metals within clusters matter as much as the total metal concentration in limiting performance. This suggested that, instead of trying to remove the impurities, we can manipulate them in a way that reduces their impact on efficiency ("defect engineering"). Further developments, such as hightemperature in situ measurements to probe defect states during processing, will provide deeper insight for additional optimization.

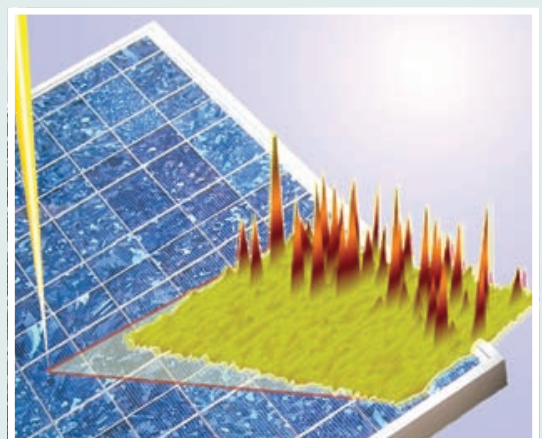

Artist's depiction of an intense beam of synchrotron light striking a solar cell and the resulting fluorescence image of the distribution of iron impurities. Courtesy of T. Buonassisi (MIT). [Buonassisi et al., Nat. Mater. 4, 676 (2005) ; Buonassisi et al., Appl. Phys. Lett. 89, 042102 (2006); Buonassisi et al., Acta Mater. 55, 6119 (2007).]

Thin-film solar cells, widely considered the next generation of photovoltaic devices, have reached efficiencies of up to $20 \%$. With high-brightness undulator radiation and a high-transmission $\mathrm{x}$-ray spectrograph, it is now possible to collect resonant inelastic soft x-ray scattering (RIXS) data in a record time (less than an hour). Together with sophisticated theoretical calculations, the observations allow a deeper understanding of the energies associated with occupied and unoccupied electronic states, giving insight into the band gap, an understanding of the excitation behavior, and element- and statespecific information about electronic wavefunctions, the most fundamental description of electrons in matter.

Small-angle x-ray scattering (SAXS) has been used to demonstrate that one can manipulate the spatial distribution of nanoparticles into specific regions of block copolymer structures using light-responsive and heat-responsive small molecules. 

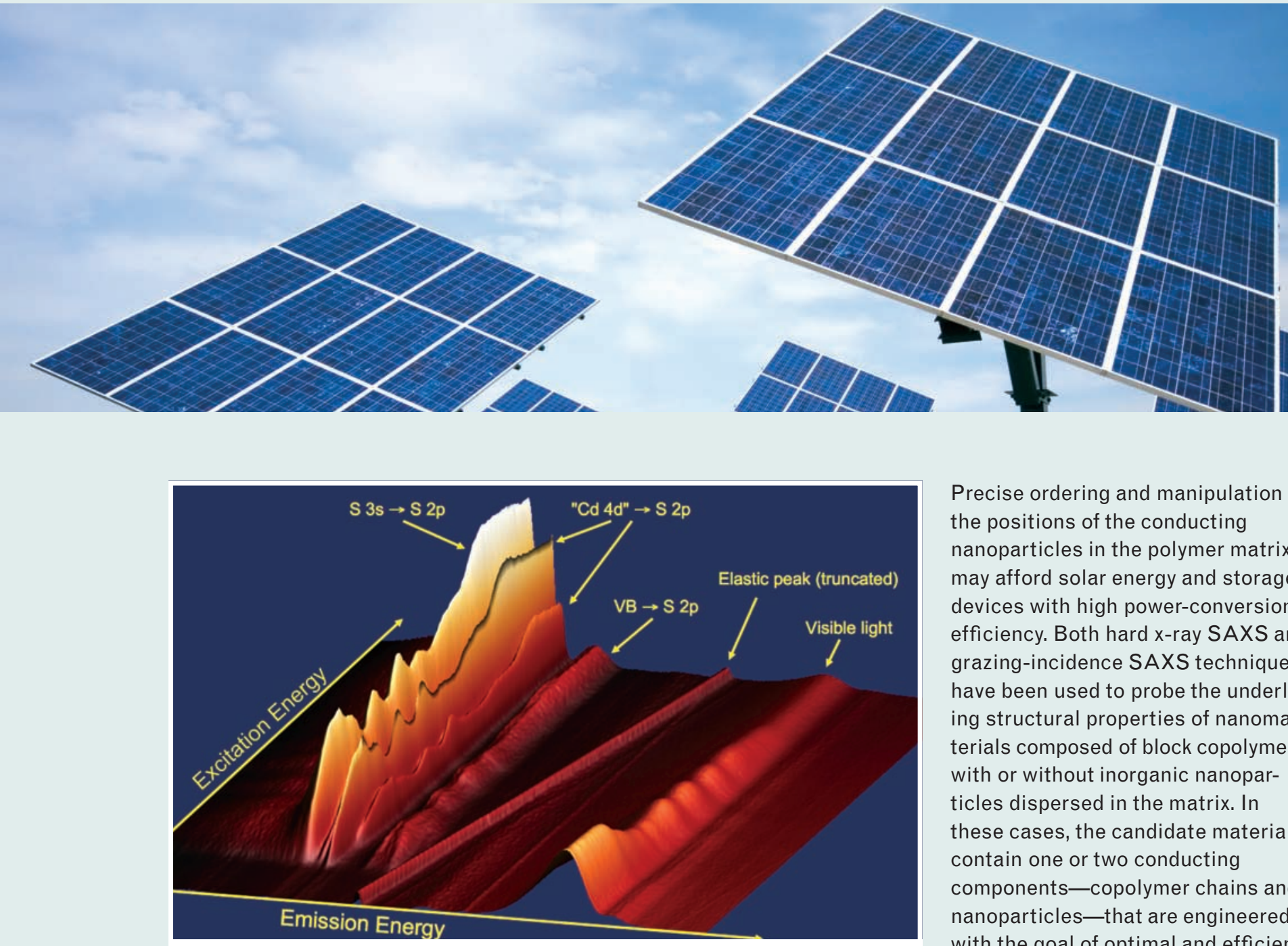

Resonant inelastic soft $x$-ray scattering map of $\mathrm{CdS}$, a central material in a variety of thin-film solar-cell devices. The map, combining information from traditional $\mathrm{x}$-ray absorption and emission spectra, gives complete information about the electronic states of a material. Courtesy of C. Heske (UNLV). [Weinhardt et al., Phys. Rev. B 79, 165305 (2009).]
Precise ordering and manipulation of the positions of the conducting nanoparticles in the polymer matrix may afford solar energy and storage devices with high power-conversion efficiency. Both hard x-ray SAXS and grazing-incidence SAXS techniques have been used to probe the underlying structural properties of nanomaterials composed of block copolymers with or without inorganic nanoparticles dispersed in the matrix. In these cases, the candidate materials contain one or two conducting components-copolymer chains and nanoparticles-that are engineered with the goal of optimal and efficient charge separation for fabricating efficient solar energy devices. 


\section{SUNLIGHT TO FUEL}

Nature has devised a remarkably diverse set of pathways to convert solar photons into chemical fuels. Learning from Nature, we hope to use plant matter as the raw material for transportation biofuels. Another avenue of research is to create an artificial photosynthetic device, made from earth-abundant materials, capable of efficiently converting sunlight and carbon dioxide into alcohol-based fuels.

Biofuels can be produced efficiently from crops with high sugar content (the sugar is converted into ethanol by yeast). Cellulose, a key component of plant cell walls, is far more abundant in plants than sugars, and plants that produce large volumes of cellulose, but no sugar (e.g., grasses) can be grown on poorer quality land, with much lower use of water and fertilizers. Cellulose is, in fact, a sugar polymer and can be broken down into sugar components, potentially providing a new, highly abundant biofuel source. The use of cellulose is hindered by its presence in cell walls as a complex mixture with hemicellulose and lignin, all of which are naturally resistant to breakdown.

Ionic liquids have emerged as promising new solvents for pretreating plant material, a process that disrupts the crystalline structure of the cellulose. To study these processes, switchgrass and corn stalks and leaves are placed into capillaries containing an ionic liquid. X-ray diffraction is then used to monitor the real-time changes of the cellulose crystalline structure, and small-angle $x$-ray scattering measures changes in the cellulose microfiber size.

These studies can be combined with infrared and Raman spectroscopy studies of changes in intermolecular hydrogen bonding. Together, these studies are designed to provide useful information on how to improve pretreatment of lignocellulosic biomass and thereby deliver cheaper biofuel.

Scientists are also exploring ways to use sunlight to convert carbon dioxide and water into fuel molecules (hydrogen, methane gas, or simple alcohols) in a single, integrated

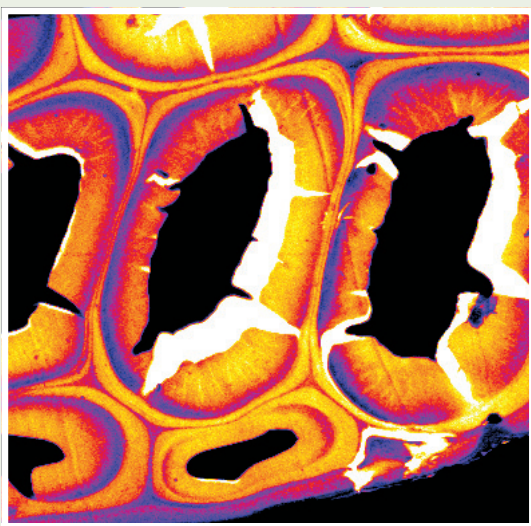

Soft $x$-ray microscopy image of cellular composition of compression wood from red spruce. The image is $40 \times 40$ microns with a spatial resolution of $100 \mathrm{~nm}$; the acquisition energy of 285 $\mathrm{eV}$ highlights the spatial distribution of lignin (purple regions). Courtesy of G. Cody (Carnegie Inst. of Washington), recorded at the National Synchrotron Light Source, 1998. 

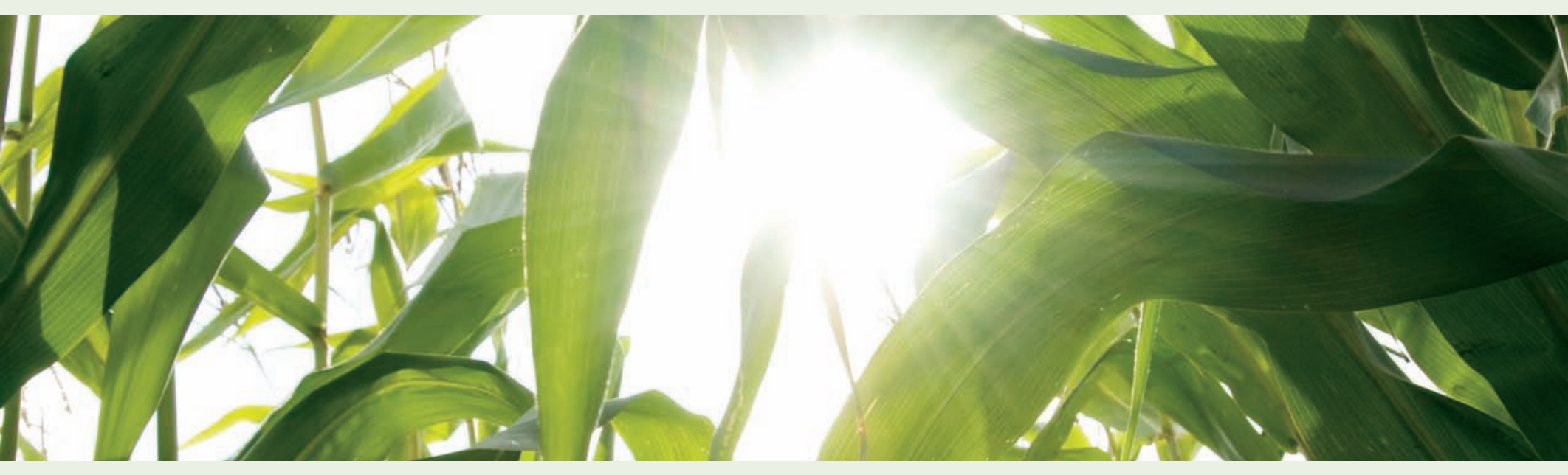

device made from earth-abundant inorganic materials (artificial photosynthesis). The electronic structures of these materials are determined in part by using synchrotron-based near-edge and extended x-ray absorption spectroscopy. Recent soft x-ray developments involving highefficiency emission spectrographs, ambient-pressure photoemission spectroscopy, and in situ measurement techniques provide a better indication of the oxidation state, symmetry, electronic structure, and charge-transfer processes in the complexes involved.

The development of light sources based on free-electron lasers is also providing new opportunities for time-resolved $x$-ray experiments that would make it possible to create movies of the breaking and formation of bonds as well as of charge-transfer

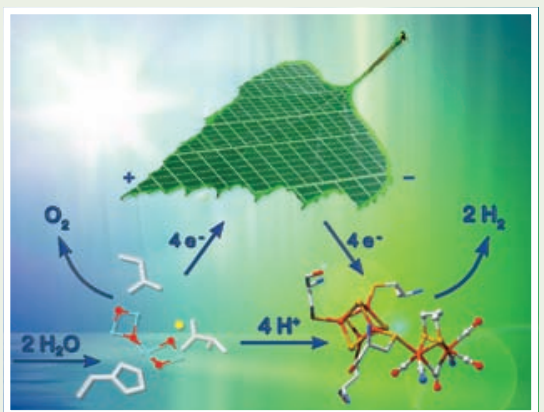

processes as they happen in time frames that range from picoseconds to femtoseconds to perhaps attoseconds. Such experiments would isolate the processes involved and reveal their evolution over time by combining the chemical and atomic specificity of $x$ rays with the use of stroboscopic pump-probe techniques.
Schematic representation of solar splitting of water. Nanotubes embedded within a membrane could act like green leaves, using incident solar radiation to split water molecules $\left(\mathrm{H}_{2} \mathrm{O}\right)$, freeing up electrons, hydrogen $\left(\mathrm{H}_{2}\right)$, and oxygen $\left(\mathrm{O}_{2}\right)$ that then react with carbon dioxide $\left(\mathrm{CO}_{2}\right)$ to produce a liquid fuel that can be used for vehicles. The result is a renewable green energy source that also helps scrub the atmosphere of excessive carbon dioxide from the burning of fossil fuels. Courtesy of W. Lubitz (Max Planck Institute). [Lubitz et al., Energy Environ. Sci. 1, 15 (2008).] 


\section{ENERGY STORAGE: BATTERIES}

Batteries give us the ability to store energy, which is important for portability (e.g., electric cars) as well as for the steady delivery of electricity using a smart grid from green but intermittent energy sources (e.g., sun and wind farms) to consumers when and where it is needed. These goals require a new generation of batteries that store more energy, recharge faster, and survive more duty cycles without degradation.

To develop better batteries, researchers must assess what is happening inside the battery materials as the batteries charge and discharge. For example, in lithium-ion batteries (used in everything from cell phones to stateof-the-art electric vehicles), researchers would like to observe how the lithium ions (which replace electrons as the charge carriers) interact with the batteries' cathodes and anodes. In the case of polymer-based batteries, researchers want to watch the block copolymers of the electrolyte change pattern or even heal themselves through annealing during heating and cooling. Problem areas also need to be addressed, such as the swelling of materials and growth of lithium dendrites into the electrolyte.

Fast small- and wide-angle x-ray scattering provides the metric needed to make nanoscale measurements

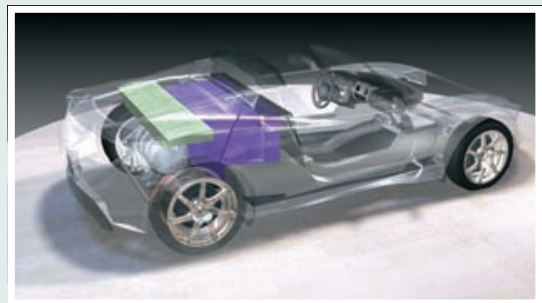

X-ray beamlines provide several tools and techniques necessary to keep up with the growth of the world's energy demands through rational design of new materials with real-world payoffs, such as car batteries with higher capacities and more duty cycles. Rendering courtesy of Tesla Motors, Inc.

of the structure of a rapidly changing battery system. Researchers have recently conducted an almost ideal experiment: entire lithium-ion batteries have been placed into the $x$-ray beam and charged and discharged in situ while SAXS measurements were made. This allowed them to quantify the nanoscale changes that occur in electrode morphology during charging and discharging. This kind of information will be invaluable in developing the next generation of battery technology.

New conductive polymers have been designed and synthesized to greatly improve the energy density of lithiumion batteries. The polymers, when made into negative electrodes with commercial silicon nanoparticles, provide both intimate contact with the nanoparticle surfaces for electrical conductivity and structural flexibility to accommodate large-volume changes during lithium-ion insertion and removal. Soft x-ray absorption spectroscopy provides direct information for a rational optimization of these materials. Synthesis of the polymers is fully compatible 

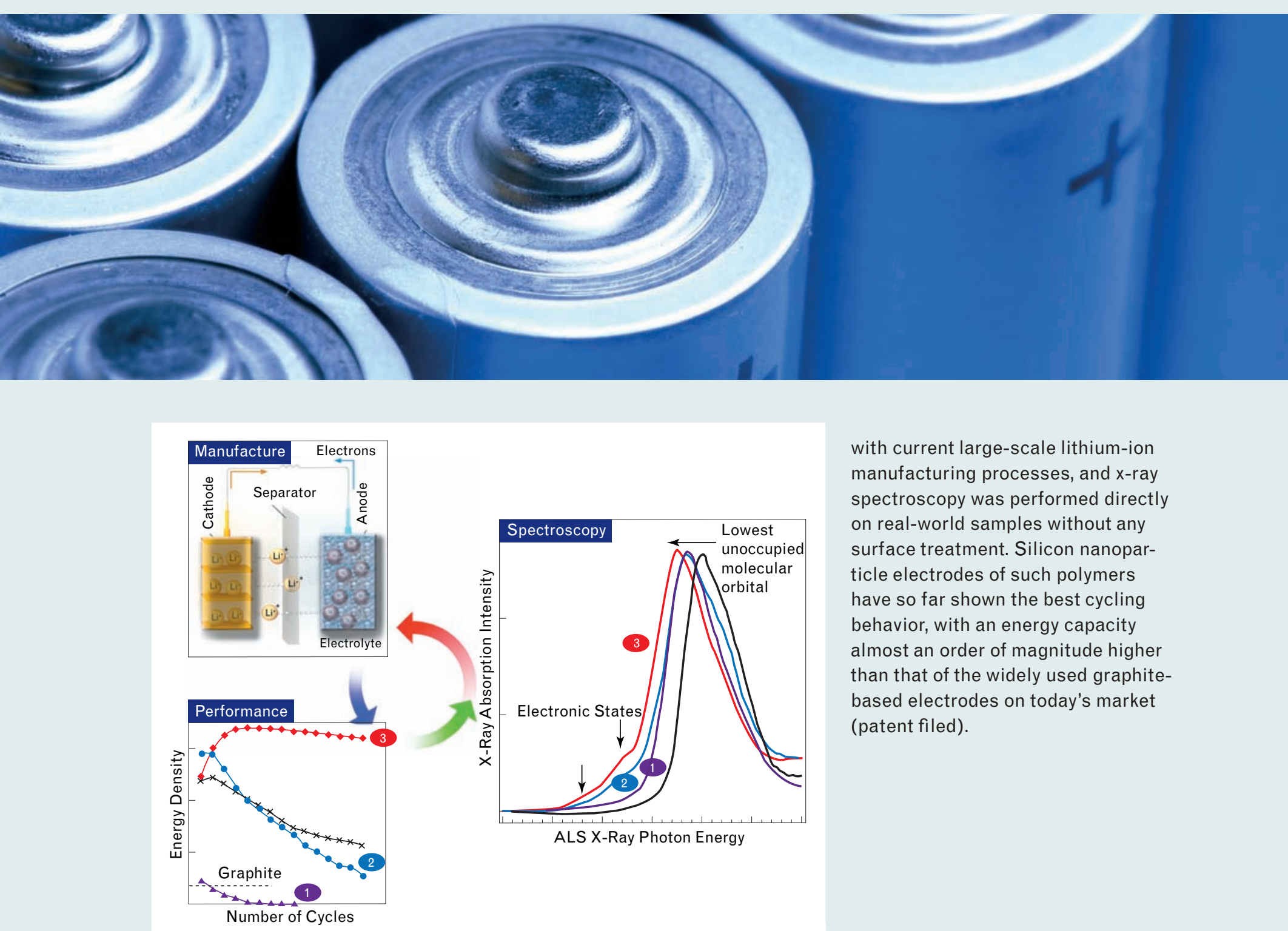

with current large-scale lithium-ion manufacturing processes, and x-ray spectroscopy was performed directly on real-world samples without any surface treatment. Silicon nanoparticle electrodes of such polymers have so far shown the best cycling behavior, with an energy capacity almost an order of magnitude higher than that of the widely used graphitebased electrodes on today's market (patent filed).

X-ray absorption spectroscopy reveals the evolution of the electronic states around the band gap in polymers with different functional groups, providing a rational basis for the design of batteries that perform up to ten times better (red curve) than what's currently available (purple curve). [G. Liu and W.L. Yang et al., unpublished (2010).] 


\section{ELECTROCHEMICAL FUEL CELLS}

When hydrogen and air are pumped into a fuel cell, electricity is generated and air and water vapor leaves. A hydrogen fuel cell can thus be classed as a zero-emission energy source provided that the hydrogen is not generated via carbon-based fuels. In situ synchrotron-based techniques and nanofabrication are providing new insight into the operation of these electrochemical devices.

Whereas batteries store energy, fuel cells require a constant fuel supplyhydrogen, for example. The hydrogen's electrons and protons are catalytically separated, and an electrolytic membrane allows the protons to travel from the cell's anode to its cathode, while forcing the electrons through an external circuit, creating an electric current.

Fuel cells that employ solid oxide membranes typically require high operating temperatures (above $650^{\circ} \mathrm{C}$ ), limiting material choices. Yttria-stabilized zirconia (YSZ) membranes and cerium oxide (ceria) electrodes have received significant attention due to properties that can, in theory, expand the electrochemically active region. However, there is little direct knowledge of local overpotential and length scales of the electrochemically active regions of such materials in opera-
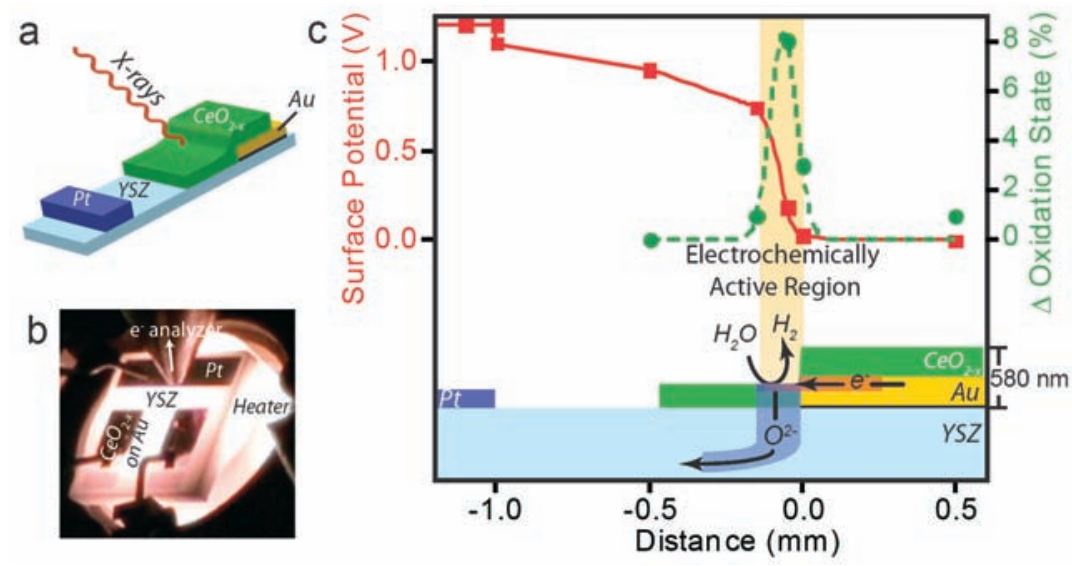

Electrochemical activity over $100 \mu \mathrm{m}$ of a ceria anode. (a) Schematic of solid oxide cell showing Pt counter electrode, Au current collector on top of a thin alumina film (black), and ceria working electrode patterned onto a YSZ single-crystal substrate. This geometry exposes the entire cell to the x-ray beam. (b)The view through the sample chamber window during cell operation at about $700^{\circ} \mathrm{C}$. (c) A ceria anode converts $\mathrm{H}_{2} \mathrm{O}$ into $\mathrm{H}_{2}$ and $\mathrm{O}^{2-}$ in a narrow region at 1.2-V cell potential. In situ APXPS reveals gradients in surface potential (red) and the shift of Ce oxidation state out of equilibrium (green) in this region. [C. Zhang et al., submitted (2010).] 


\section{$\mathrm{CO}_{2}$ CAPTURE AND SEQUESTRATION}

Capturing carbon dioxide $\left(\mathrm{CO}_{2}\right)$ gas from stationary sources such as power stations, and then pumping it underground for storage in permeable rock is a potential way to avoid or mitigate "greenhouse gas" effects on climate. What materials are best at efficiently capturing the $\mathrm{CO}_{2}$ ? What happens to the $\mathrm{CO}_{2}$ when underground?

The separation of $\mathrm{CO}_{2}$ from other emitted gases requires materials exhibiting exactly the right adsorption or diffusion properties. Metal-organic frameworks (MOFs) are molecular cages with channels or pores whose sizes can be tailored to selectively trap $\mathrm{CO}_{2}$ gas. Perhaps the most powerful tool available for analyzing the structure of MOFs is single-crystal $x$-ray diffraction. Combining this technique with an in situ gas environmental cell can reveal how porous MOFs store gas molecules such as $\mathrm{CO}_{2}$. Various soft x-ray spectroscopies can also reveal the electronic structures of MOFs and possibly of the gas species as well, with the aim of better understanding the interactions between these materials. This would complement studies of crystal structure and might ideally even look at the same materials. The combination of electronic and crystal

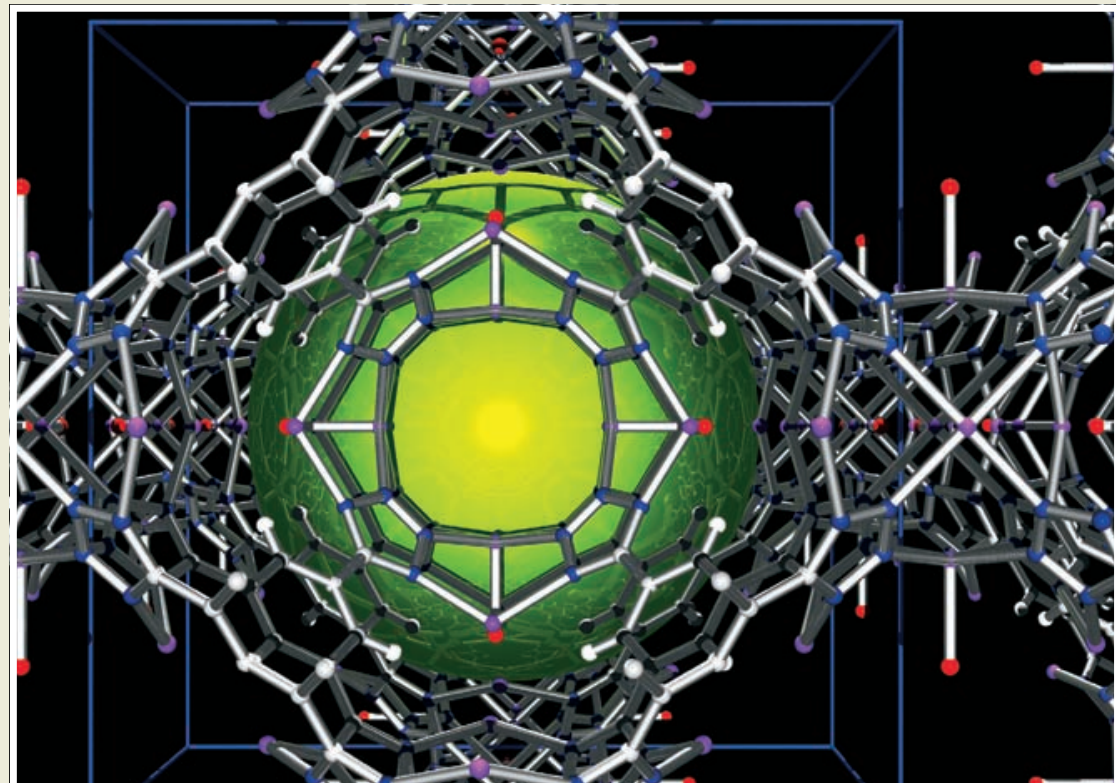

Illustration of a metal-organic framework (MOF) structure. Metal compounds are connected via organic-molecule bridges into a lightweight scaffolding (gray sticks) with large voids (yellow sphere) that can accomodate "guest" gas molecules such as $\mathrm{CO}_{2}$. Illustration by Christine Beavers (ALS). [Dinca et al., J. Am. Chem. Soc. 128, 16876 (2006).] 

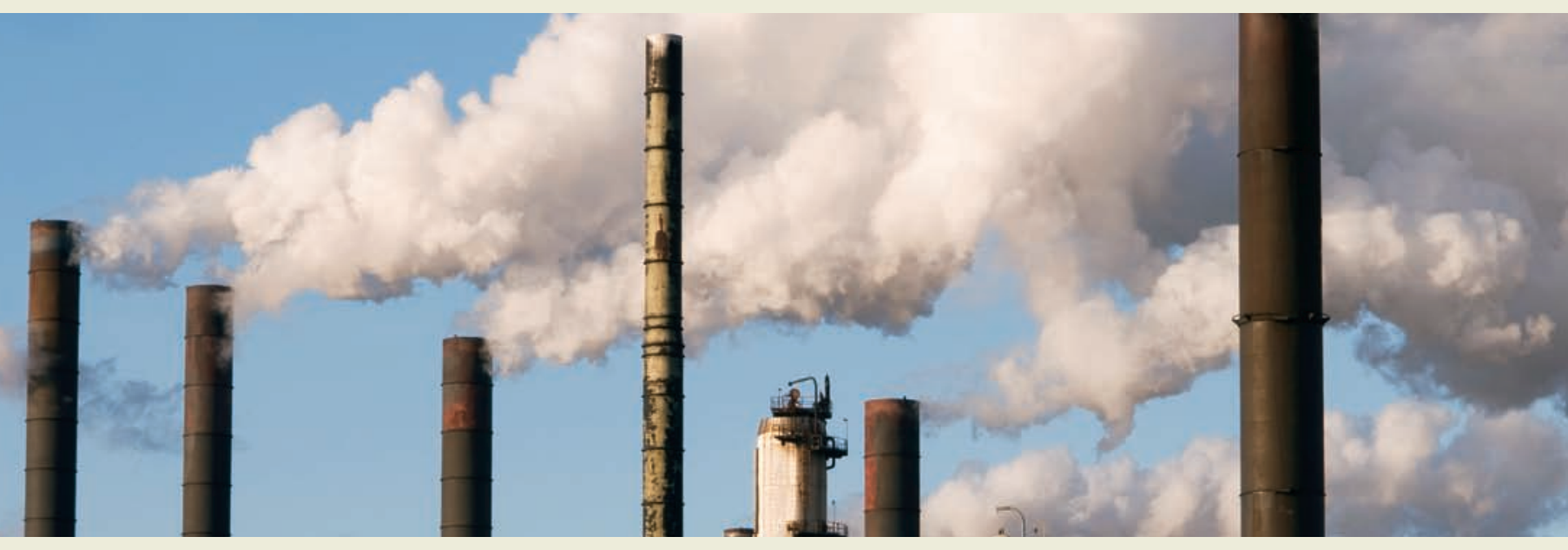

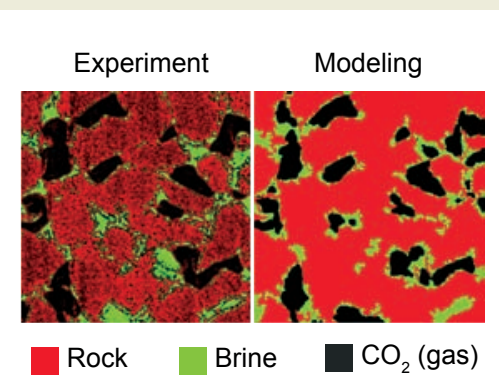

Comparison between microtomography data (left) and modeling (right) for the displacement of brine from sandstone by $\mathrm{CO}_{2}$ gas. The black speckles in the experimental data represent noise. [Silin et al., American Geophysical Union Fall Meeting 2008.]

structure information would provide more data to compare with theoretical models of gas interactions with porous host materials.
When injected into underground rock structures, the flow properties of $\mathrm{CO}_{2}$ are dominated by the innumerable fluid/fluid exchange interactions within the micron-sized pores of the rock. Understanding these interactions is the key to predicting the behavior of underground $\mathrm{CO}_{2}$ reservoirs. Using synchrotron light, we are able to look inside rocks with three-dimensional x-ray microtomography to see how fluids flow through permeable rock, displacing existing liquids. These experiments yield the data that will confirm the theoretical models being developed that will in turn be used to predict the properties of large $\mathrm{CO}_{2}$ underground reservoirs.

The interaction of $\mathrm{CO}_{2}$ with rocks, soil, ice, sea water, and aerosols plays an important part in the $\mathrm{CO}_{2}$ chemistry of Earth's atmosphere. In particular, chemical processes at the interfaces between solid, liquid, and vapor determine the uptake and release of $\mathrm{CO}_{2}$. Ambient-pressure $x$-ray photoelectron spectroscopy (APXPS) allows the investigation of these interfaces under realistic conditions of relative humidity and $\mathrm{CO}_{2}$ partial pressure. Current projects include the investigation of the uptake of $\mathrm{CO}_{2}$ by mineral surfaces (e.g. $\mathrm{MgO}$ ) as a function of relative humidity, where in particular the formation and possible removal of selfpassivating carbonate layers (which limit $\mathrm{CO}_{2}$ uptake by mineral surfaces) is of interest [Hendrik Bluhm (Berkeley Lab) et al.]. 


\section{COMBUSTION}

Fossil fuels, biofuels, and synthetic alcohol-based fuels all have different combustion processes. After more than 150 years of study, combustion is well understood in terms of average energy output, high-concentration intermediates, and major products. However, it is necessary to understand flame chemistry at the partsper-million level if we are to further improve efficiency and control pollution.

As fuel burns and transforms into its final products, it goes through an intricate network of thousands of reaction pathways with hundreds of short-lived transient molecules (reaction intermediates). These often include lung-damaging particulates like soot, smog-causing nitrogen oxides, and other pollutants. Detailed mathematical models have been developed to help improve combustion efficiency and reduce pollution. However, the accuracy of these models depends on knowing all the chemical species involved and having quantitative values for the reaction constants that describe how the reactions proceed. As a result, many important rate constants have never been measured directly, nor have all the species included in mathematical flame models been directly observed.

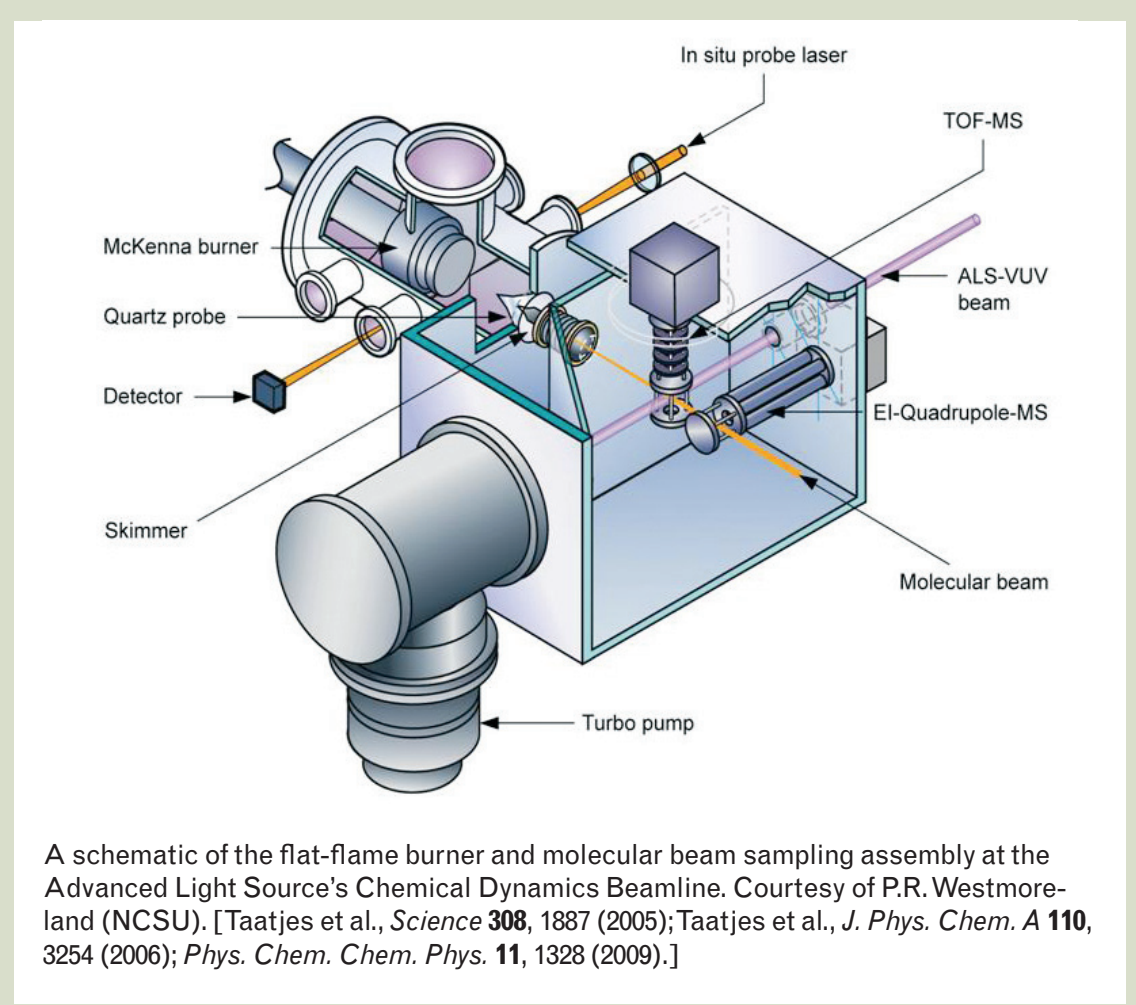


For those studying flame chemistry and the properties of combustion intermediates by means of molecular beam mass spectrometry, the use of tunable vacuum ultraviolet light from a synchrotron to photoionize the beam for mass spectrometry makes for a powerful technique capable of differentiating between isomers with the same molecular weight and composition. Using such a technique, researchers discovered that a class of compounds known as enols (ethenol or vinyl alcohol is the simplest of these), only recently observed as a combustion intermediate, is in fact widely present in flames burning a variety of fuels, including commercial blends, such as gasoline. Combustion modelers will have to take this new finding to heart, as will those studying other forms of hydrocarbon oxidation, including those involving synthetic and biofuels.

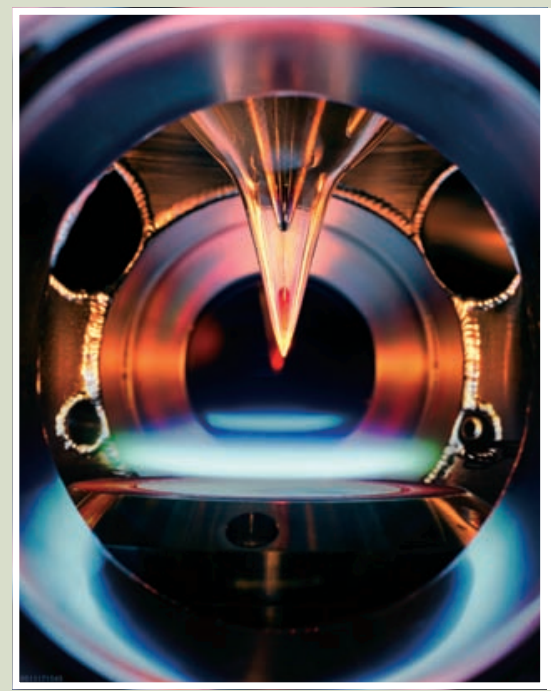

The luminous zone of the flat flame has the typical blue-violet or blue-green color associated with chemiluminescence from electronically excited $\mathrm{CH}$ and $\mathrm{C}_{2}$. The gases produced by the flame are sampled through a small aperture in a quartz cone. These gases can then be analyzed by synchrotron-photoionization mass spectrometry, which has revealed previously undetected combustion intermediates. Photo by Linda Hadley (Sandia National Laboratories). 


\section{CATALYSIS}

Catalysts—substances that speed up chemical reactions without being consumed-play a central role in technologies relevant to energy and the environment. Because of their importance, researchers are working to develop cheaper and smarter catalysts that are fine-tuned to accelerate reactions that, for example, drive fuel-refinement techniques, feed hydrogen fuel cells, or sweep toxins from emissions.

The catalysts used in chemical processes often consist of nanoscale metal or metal oxide particles dispersed on a surface. While these particles are the active elements of the catalyst, their overall performance depends not only on their size and composition but also on their interactions with the surface, reactants, products, and environment (gaseous atmospheres at high temperatures). Probing this chemical soup in real time under realistic reaction conditions is a tall order, and such catalysts could previously be observed only before and after, but not during, a reaction. In some cases even the catalytically active chemical species is not known.

By combining scanning transmission x-ray microscopy (STXM) with a reaction chamber adapted from electron microscopy, we can identify
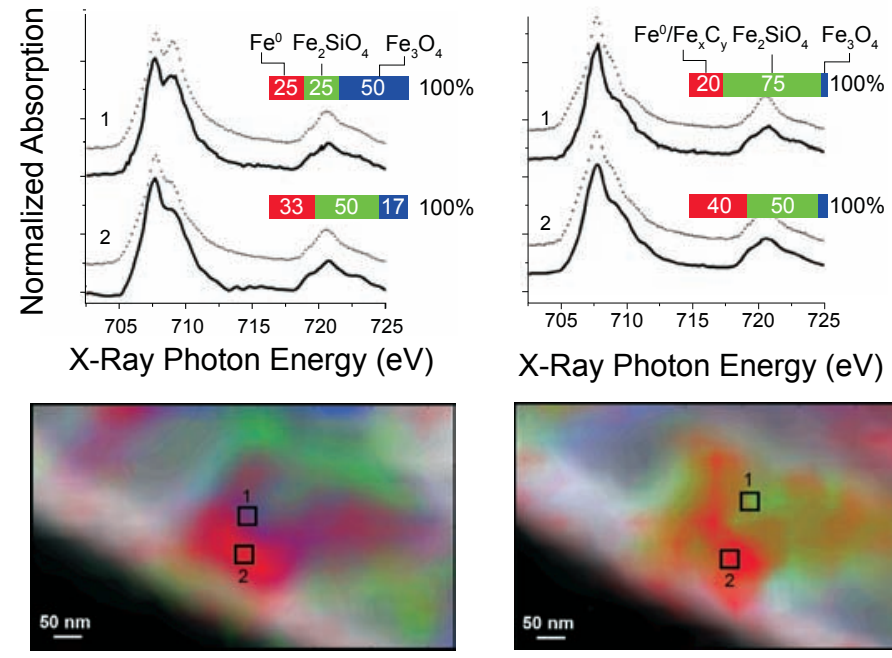

\section{X-Ray Photon Energy (eV)}

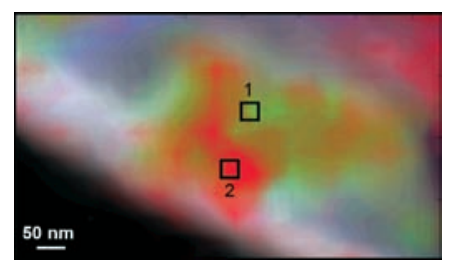

Tracking changes in an iron-based catalyst. Top left: Iron spectra taken before catalysis from two regions (1 and 2) of the catalyst material. Top right: Spectra taken after catalysis. Dotted lines are calculated from reference spectra. Bar graphs show the relative contributions of different iron phases at the sampling points. Bottom: Iron species contour maps generated from the spectra taken at each pixel. The colors are keyed to the bar graphs above. Courtesy of F.M.F. de Groot (Utrecht University). [de Smit et al., Nature 456, 222 (2008).] 


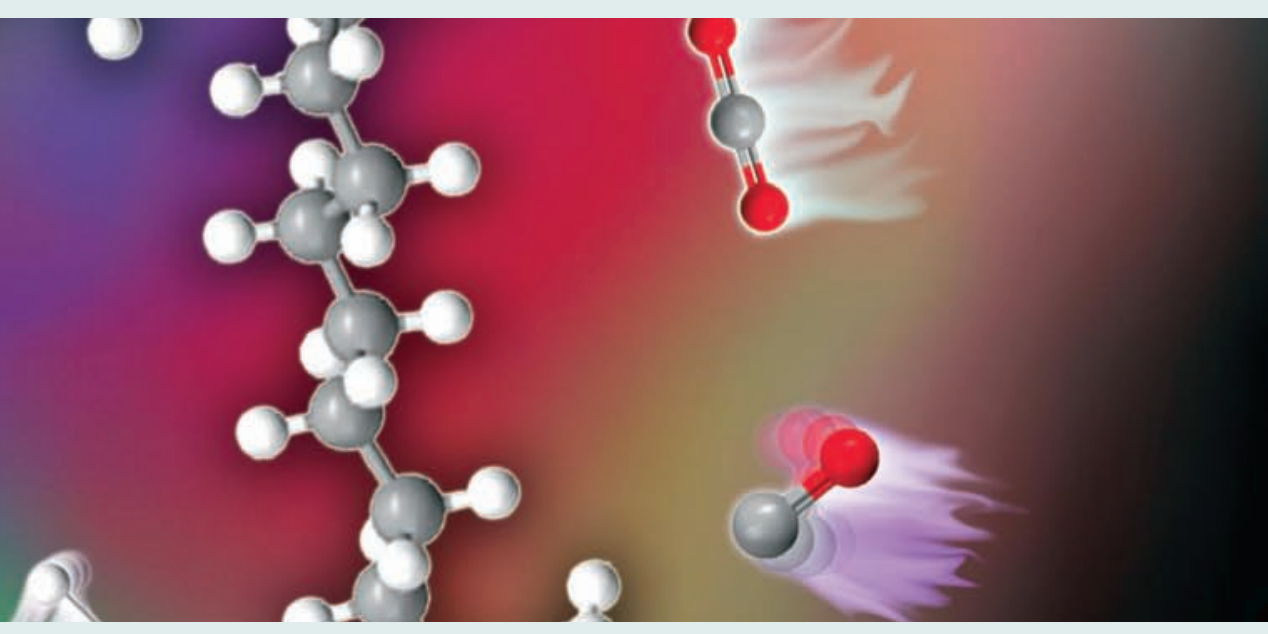

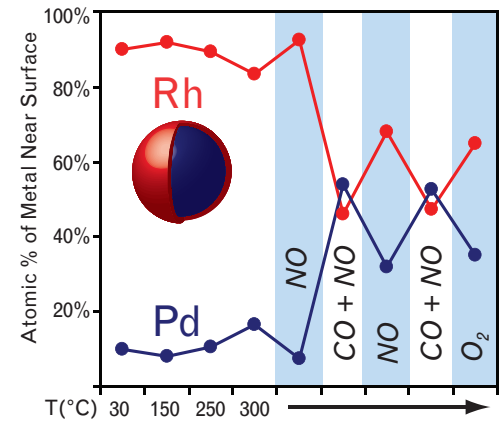

Bimetallic nanoparticles are synthesized with a rhodium-rich shell. Under reaction conditions, the composition of the outer shell is determined by the temperature and the composition of gases surrounding the nanoparticles. Courtesy of G.A. Somorjai (UC Berkeley). [Tao et al., Science 322, 932 (2008).] the chemical species present and see their distribution on the nanoscale. Such measurements demonstrate that STXM can provide details about the morphology and composition of complex catalytic systems under realistic conditions. When developed further, this new tool may give chemists the ability to design and tailor catalysts for maximum selectivity and efficiency in a wide range of chemical processes.

Some of the most active and selective catalysts are bimetallic nanoparticles, with the two metals variously distributed between the particle core and shell. In situ studies using ambient-pressure $x$-ray photoelectron spectroscopy have been used to demonstrate that bimetallic catalysts can undergo profound structural and chemical changes in response to reactive environments at ambient pressures. With tunable synchrotron light, it is possible to create depth profiles of particle composition and chemical state. This work is a first step toward designing "smart" catalysts whose structures change advantageously depending on the reaction environment. 
